Brazilian Journal

of Chemical

ISSN 0104-6632

Engineering

\title{
DETERMINATION OF SOLUBILITY PARAMETERS AND THERMODYNAMIC PROPERTIES IN HYDROCARBON-SOLVENT SYSTEMS BY GAS CHROMATOGRAPHY
}

\author{
E. Díaz , A. Cazurro, S. Ordóñez, A. Vega and J. Coca \\ Department of Chemical Engineering and Environmental Technology, \\ University of Oviedo, Fax: (34) 985103 434, Julián Clavería s/n, 33006, Oviedo, Spain \\ E-mail: eva@genio.quimica.uniovi.es
}

(Received: October 4, 2005 ; Accepted: December 20, 2006)

\begin{abstract}
Gas chromatography used to calculate the specific retention volume of several hydrocarbons in different chromatographic liquid phases (Squalane, Carbowax-400, Carbowax-1500, Carbowax-4000, Amine220, Dinonyl phthalate, Tributyl phosphate and Trixylenyl phosphate). Some thermodynamic parameters, such as enthalpy of sorption and Flory-Huggins parameters relating the interaction between liquid phases and solutes, were also calculated from the determined retention volumes. Liquid phase solubility parameters of Squalane, Carbowax-400, Carbowax- 1500 and Carbowax-4000 at $80{ }^{\circ} \mathrm{C}$ as well as the polar and apolar components were calculated too. A new model was proposed to correlate polar contribution to the solubility parameter of a liquid phase with the specific retention volume of a solute in this liquid phase.

Keywords: Gas chromatography; Solubility parameter; Specific retention volume; Polar component; FloryHuggins interaction parameters.
\end{abstract}

\section{INTRODUCTION}

Gas chromatography is an appropriate tool for characterizing bulk solvent or surface interactions of solutes with substances that can be coated as liquid films (or particle layers) on chromatographic supports or the walls of open tubular columns (Abraham et al., 1999). Only small amounts of material are required and physicochemical properties can be determined over a wide temperature range when allowed by the physical properties of the materials.

The chromatographic experiment provides a retention parameter, such as specific retention volume, that requires a model to express the results in a suitable form for interpretation of material properties. The solubility parameter concept is widely used because it provides a rough and ready approximation of solubility behaviour. It may be used directly, as in the classification of stationary phases, or indirectly to obtain quantitative structurebehaviour relationships, such as the relationship between polarity and relative volatility (Everson and Van der Merwe, 1998), which could be very useful in separating two solutes with very close boiling points. This is the purpose of extractive distillation, a separation process often considered as an alternative to conventional distillation.

In extractive distillation, a high boiling point solvent is introduced into the column in order to produce an alteration of the key relative volatility of each component (Vega et al., 1997). Owing to large deviations from ideality of the extractive distillation systems, the best solvent cannot generally be

*To whom correspondence should be addressed 
selected from pure components properties only. At least in a preliminary stage, the appropriate solvent for a given separation can be selected based on selectivity at infinite dilution, defined as the ratio of the activity coefficients of both components at infinite dilution in the solvent. An efficient extractive distillation solvent must have not only high selectivity, but also sufficient capacity or solvency for to have a good performance in the distillation process. The pattern of selectivity-solvency can aid in the reliable choice of solvents having the best balance of these properties, although other factors, such as solvent cost and availability, solvent thermal and chemical stability, corrosion, etc, must be taken into account (Vega et al., 1997). The infinite dilution activity coefficient for a given solute can be used to characterize the solvent capacity or solvency (Deal and Derr, 1964).

The main purpose of this work is to obtain the solubility parameter for different combinations of hydrocarbons and liquid phases, a topic that has been extensively studied since the seventies (Letcher et al., 2001, 2004). The aim of this work is to develop a simple model for obtaining a correlation between the retention volume for hydrocarbon series and the polar contribution to the solubility parameter.

\section{THEORY}

The retention data determined by chromatography experiments were used to calculate the thermodynamic functions of solute dissolution in the solvents as well as solubility parameters.

The retention volume of the solute depends on its partition between the stationary and mobile phase and is an indication of the bonding strength between the solute molecule and the liquid phase. It is defined as the volume of dry carrier gas necessary to elute the solute sample at $0{ }^{\circ} \mathrm{C}$ per gram of stationary liquid phase. The specific retention volumes were determined by the following equation:

$\mathrm{V}_{\mathrm{g}}^{0}=\left(\mathrm{t}_{\mathrm{R}}-\mathrm{t}_{\mathrm{d}}\right)\left(\frac{273.15 \cdot \mathrm{F}}{\mathrm{T}_{\mathrm{o}} \mathrm{w}_{\mathrm{s}}}\right)\left(\frac{\mathrm{p}_{\mathrm{o}}-\mathrm{p}_{\mathrm{w}}}{\mathrm{p}_{\mathrm{o}}}\right) \mathrm{j}$

where $t_{R}$ is the retention time in minutes; $t_{d}$, the dead time ( $\mathrm{min}) ; \mathrm{F}$, the carrier gas flow-rate $(\mathrm{ml} / \mathrm{min}) ; \mathrm{T}_{\mathrm{o}}$, the flow meter temperature $(\mathrm{K}) ; \mathrm{w}_{\mathrm{s}}$, the mass of the stationary phase $(\mathrm{g}) ; \mathrm{p}_{\mathrm{o}}$, the outlet column pressure $(\mathrm{Pa}) ; \mathrm{p}_{\mathrm{w}}$, the vapor pressure of water at the flow meter temperature $(\mathrm{Pa})$; and $\mathrm{j}$, the James-Martin compressibility factor (which takes into account the pressure profile in the column) defined as

$\mathrm{j}=\frac{3}{2}\left[\frac{\left(\mathrm{p}_{\mathrm{i}} / \mathrm{p}_{\mathrm{o}}\right)^{2}-1}{\left(\mathrm{p}_{\mathrm{i}} / \mathrm{p}_{\mathrm{o}}\right)^{3}-1}\right]$

where $p_{i}$ is the pressure at the beginning of the chromatographic column.

The retention volumes are related to the activity coefficients. These coefficients at infinite dilution are an important property, which is used for the selection of selective solvents (extraction and extractive distillation) and for the reliable design of thermal separation processes. More concretely, the influence of the solvent is usually quantified in terms of its so-called selectivity, which is defined as the ratio of the activity coefficients of two components in the presence of the solvent. The activity coefficients of solute 1 at infinite dilution in solvent $2, \gamma_{1}^{\infty}$, were determined from the specific retention volume, $\mathrm{V}_{\mathrm{g}}^{0}$, by the following equation, (Weidlich et al., 1987; Vega and Coca, 1991):

$\ln \gamma_{1}^{\infty}=\ln \left(\frac{273.15 \mathrm{R}}{\mathrm{V}_{\mathrm{g}}^{0} \mathrm{M}_{2} \mathrm{P}_{1}^{0}}\right)-\frac{\mathrm{P}_{1}^{0} \mathrm{~B}_{11}}{\mathrm{RT}}$

where $\mathrm{M}_{2}$ is the molecular weight of the liquid phase; $\mathrm{P}_{1}{ }^{\circ}$, the saturated vapour pressure of the pure solute; $\mathrm{B}_{11}$, the second virial interaction coefficient of the solute; and R, the gas constant. Vapour pressures were calculated using the Antoine equation, and $B_{11}$ was estimated from the BeattieBridgeman correlation (Alessi et al., 1978):

$$
\mathrm{B}_{11} / \mathrm{V}_{\mathrm{c}}=0.461-1.158\left(\mathrm{~T}_{\mathrm{c}} / \mathrm{T}\right)-0.503\left(\mathrm{~T}_{\mathrm{c}} / \mathrm{T}\right)^{3}
$$

where $V_{c}$ and $T_{c}$ are the critical volume and temperature of the solute, respectively, values which were obtained from the literature (ESDU, 1987).

Likewise, also related to the specific retention volume is the molar heat (entalphy), $\Delta \mathrm{H}^{\mathrm{S}}{ }_{1}$, of sorption of the probe in the stationary phase. This parameter relates the absolute temperature $(\mathrm{K})$ and the specific retention volume, in accordance with the following equation, (Kaya et al., 2002):

$$
\Delta \mathrm{H}_{1}^{\mathrm{S}}=-\mathrm{R} \frac{\partial \ln \mathrm{V}_{\mathrm{g}}^{0}}{\partial(1 / \mathrm{T})}
$$


The activity coefficient is commonly expressed as the contribution of two effects: the thermal contribution, $\gamma_{\mathrm{t}}^{\infty}$, resulting from molecular interactions of the compounds, and the athermal contribution, $\gamma_{\mathrm{a}}^{\infty}$, associated with the difference in size between the solute and solvent molecules:

$\ln \gamma_{1}^{\infty}=\ln \gamma_{t}^{\infty}+\ln \gamma_{a}^{\infty}$

The interaction parameter, $\chi^{\infty}$, is related with $\ln$ $\gamma_{1}^{\infty}$ by subtracting the Flory-Huggins size correction:

$\chi^{\infty}=\ln \gamma_{1}^{\infty}-\ln \left(\frac{1}{r}\right)-\left(1-\frac{1}{r}\right)$

where $r$ is approximated as the molar volume ratio of solvent to solute.

The size correction is usually taken as the value of $\ln \gamma_{a}^{\infty}$. The interaction parameter redefined as "residual" free energy involves the enthalpy and entropy effects

$\chi^{\infty}=\chi_{\mathrm{H}}^{\infty}+\chi_{\mathrm{S}}^{\infty}$

and from the regular solution theory

$\chi_{\mathrm{H}}^{\infty}=\left(\mathrm{V}_{1}^{0} / \mathrm{RT}\right)\left(\delta_{2}-\delta_{1}\right)^{2}$

The solubility parameter is widely used to give a rough and ready approximation of solubility behaviour. The solubility parameter of the solute, $\delta_{1}$, is the square root of the cohesive energy density of a compound and thus is a measure of the strength of its intermolecular forces (Hildebrand and Scott, 1962). It is related to the enthalpy of vaporization, $\Delta \mathrm{H}^{\text {vap }}$, and the molar volume, $\mathrm{V}_{1}{ }^{\circ}$, by

$\delta_{1}=\sqrt{\frac{\Delta \mathrm{E}}{\mathrm{V}_{1}^{0}}}=\sqrt{\frac{\Delta \mathrm{H}^{\text {vap }}-\mathrm{RT}}{\mathrm{V}_{1}^{\mathrm{o}}}}$

Substituting Eq. (8) into Eq. (9), the solubility parameter of the stationary phase, $\delta_{2}$, can be calculated

$\left(\frac{\delta_{1}^{2}}{\mathrm{RT}}-\frac{\chi^{\infty}}{\mathrm{V}_{1}^{0}}\right)=\left(\frac{2 \delta_{2}}{\mathrm{RT}}\right) \delta_{1}-\left(\frac{\delta_{2}^{2}}{\mathrm{RT}}+\frac{\chi_{\mathrm{S}}^{\infty}}{\mathrm{V}_{1}^{0}}\right)$

A plot of the left side of Eq. (11) against the probe solubility parameter, $\delta_{1}$, a straight line with a slope of $2 \delta_{2} / \mathrm{RT}$ is obtained. This approach has been applied to a wide range of materials including polymers (Hamedi and Danner, 2001), organic pollutants (Voelkel and Kopczynski, 1998), chromatographic stationary phases (Vega and Coca, 1991) and biological materials (Pena et al., 2000) as well as electrokinetic chromatographies (Liu et al., 1999).

The effect of polar interactions can be approached by dividing the solubility parameter into thr different contributions. The interaction energies could be considered as additive contributions from dispersion and polar interactions:

$\delta^{2}=\lambda^{2}+\tau^{2}$

where $\lambda$ represents the non-polar (due to dispersive forces between molecules and stationary phase) and $\tau$, the polar component of the solubility parameter. The polar component, $\tau$, may be subdivided into an electrostatic part (due to electron density of the chemical structure), $\delta_{\mathrm{p}}$, and a hydrogen bonding part, $\delta_{\mathrm{H}}$. Probes interacting only with dispersive intermolecular forces (i.e. n-alkanes) should give a linear relation when plotted in accordance with Eq. (11). However, solutes with other contributions to their retention will deviate from the line, with the degree of deviation being a measure of the strength of the polar components.

\section{EXPERIMENTAL SECTION}

A Hewlett-Packard gas chromatograph 5750 equipped with a thermal conductivity detector was used for this study. Stainless steel columns $(1.5 \mathrm{~m} \times$ $5.3 \mathrm{~mm}$ i.d.) were used for the measurements, which were made using helium as the carrier gas. The solid support used was a Chromosorb P-AW (60/80 mesh) loaded with $15 \%(\mathrm{w} / \mathrm{w})$ of the liquid phase under investigation. The packing material was prepared by coating with a solution of the liquid phase in a solvent (dichloromethane) in a two-neck open flask and boiling the solution for 30 minutes with total reflux. The effect of bubbling was enough to obtain an appropriate impregnation (Vega and Coca, 1991). Then, the solvent (dichloromethane) was evaporated from the same flask. Packing was kept for 12 hours at a fixed temperature (lower than the boiling temperatures of the liquid phases - Table 1 - in order to avoid their evaporation) and purged with a slow stream of nitrogen. After this, the column was prepared and mounted in the chromatographic oven. 
The flow rate was $30 \mathrm{~cm}^{3} / \mathrm{min}$, and it was measured using a calibrated soap bubble flow meter.

Preliminary tests with variable amounts of sample showed that up to $2 \mu \mathrm{L}$, the retention times obtained did not depend on sample volume. For this reason, the injection sample was always less than $2 \mu \mathrm{L}$. The elution peaks were only slightly asymmetric, which is a good indication of infinite dilution conditions (Conder, 1969).
Solutes (hydrocarbons) were reagent grade from Fluka; purities and other information for these compounds are given in Table 1. The liquid phases used as well as their manufacturers are listed in Table 2. In order to minimize the bleeding of the column, a presaturator containing packing coated with the liquid phase was connected between the carrier gas regulator and the injection point to saturate the carrier gas with the liquid phase.

Table 1: Physical properties of solutes used in the present work

\begin{tabular}{|c|c|c|c|}
\hline Solute & Mw (g/mol) & BP $\left({ }^{\circ} \mathrm{C}\right)$ & Purity \\
\hline n-hexane & 86.18 & 68.9 & $>98.5 \%$ \\
\hline n-heptane & 100.20 & 98.5 & $>99.5 \%$ \\
\hline n-octane & 114.23 & 125.7 & $>99.8 \%$ \\
\hline n-nonane & 128.60 & 150.8 & $>99.8 \%$ \\
\hline n-decane & 142.28 & 174.2 & $>99.8 \%$ \\
\hline 1-hexene & 84.16 & 64.0 & $>99 \%$ \\
\hline 1-heptene & 98.19 & 94.0 & $99 \%$ \\
\hline 1-octene & 112.21 & 122.0 & $>99.8 \%$ \\
\hline 1-nonene & 126.24 & 146.4 & $>99.5 \%$ \\
\hline 1-decene & 140.27 & 167.0 & $>99.5 \%$ \\
\hline benzene & 78.11 & 80.3 & $>99 \%$ \\
\hline toluene & 92.14 & 110.8 & $>99.7 \%$ \\
\hline ethylbenzene & 106.17 & 136.3 & $>99.5 \%$ \\
\hline propylbenzene & 120.19 & 159.0 & $>99 \%$ \\
\hline isopropylbenzene & 120.19 & 152.0 & $98 \%$ \\
\hline cyclohexane & 84.16 & 80.9 & $>99.5 \%$ \\
\hline methylcyclohexane & 98.19 & 101.0 & $>99 \%$ \\
\hline o-xylene & 106.17 & 144.0 & $>99.5 \%$ \\
\hline m-xylene & 106.17 & 139.3 & $>99 \%$ \\
\hline p-xylene & 106.17 & 138.4 & $99 \%$ \\
\hline styrene & 104.15 & 146.0 & $>99 \%$ \\
\hline
\end{tabular}

Table 2: Liquid phases studied in this work

\begin{tabular}{|l|c|c|}
\hline \multicolumn{1}{|c|}{ Stationary phase } & Manufacturer & Purity \\
\hline Dinonyl phthalate (DP) & Sigma-Aldrich & $>99 \%$ \\
Squalane (SQ) & Fluka & $99 \%$ \\
Carbowax 400 (C-400) & Panreac & $>95 \%$ \\
Carbowax 1500 (C-1500) & Panreac & $>95 \%$ \\
Carbowax 4000 (C-4000) & Panreac & $>95 \%$ \\
Amine 220 (A-220) & Alltech & $>99 \%$ \\
Tributyl phosphate (TBP) & Sigma-Aldrich & $>99 \%$ \\
Trixylenyl phosphate (TXP) & Graco & $>98 \%$ \\
\hline
\end{tabular}

\section{RESULTS AND DISCUSSION}

The specific retention volumes, $\mathrm{V}_{\mathrm{g}}^{0}$, of the solutes calculated in accordance with Eq. (1) and activity coefficients at infinite dilution, $\gamma^{\infty}$, calculated in accordance with Eq. (3) are given in Tables 3 to 10. As shown in Tables 3 to 10 , values of $\mathrm{V}_{\mathrm{g}}{ }^{0}$ increase with the molecular weight of each group of solvents with an elution order from a smaller to a larger number of carbon atoms for each homologous solute series.
The behaviour of alkenes and n-alkanes is similar, as can be observed in Figs. 1 and 2. In both cases the $\mathrm{V}_{\mathrm{g}}{ }^{0}$ parameter decreases with operation temperature (reasonable, considering that this parameter is characterised by an exothermic equilibrium process) and increases as the solute molecular weight increases (higher molecular weight results in a higher boiling point and a higher affinity for condensed phases).

For the polarity of the liquid phases, this parameter decreases in the order C-400 > C-1500 > 
C-4000 > TBP $>$ TXP $>$ A-220 $>$ DP $>$ SQ, so Carbowax-1500 was taken as representative of high polarity solvents, whereas Amine-220 was been taken as an example of low polarity solvents. Hence, it can be observed that both, n-alkanes and alkenes have higher affinity for the less polar solvent. In the case of alkenes this result and the likeness of the general behaviour between the alkenes and n-alkanes studied, lead one to think that the absorption properties of these alkenes are controlled by the bulk organic structure, rather than by the presence of the double bond.

Table 3: Specific retention volume $(\mathrm{ml} / \mathrm{g})$ and activity coefficients at infinite dilution calculated for Dinonyl phthalate at different temperatures

\begin{tabular}{|c|c|c|c|c|c|c|c|c|}
\hline \multicolumn{9}{|c|}{ Dinonyl phthalate } \\
\hline \multirow[t]{2}{*}{ Solute } & \multicolumn{2}{|c|}{$\mathrm{T}=90^{\circ} \mathrm{C}$} & \multicolumn{2}{|c|}{$\mathrm{T}=100^{\circ} \mathrm{C}$} & \multicolumn{2}{|c|}{$\mathrm{T}=120^{\circ} \mathrm{C}$} & \multicolumn{2}{|c|}{$\mathrm{T}=130^{\circ} \mathrm{C}$} \\
\hline & $\mathbf{V}_{\mathrm{g}}{ }^{\mathbf{0}}$ & $\gamma^{\infty}$ & $\mathbf{V}_{\mathrm{g}}{ }^{0}$ & $\gamma^{\infty}$ & $\mathbf{V}_{\mathrm{g}}{ }^{\mathbf{2}}$ & $\gamma^{\infty}$ & $\mathbf{V}_{\mathbf{g}}^{\mathbf{0}}$ & $\gamma^{\infty}$ \\
\hline n-hexane & 25.8 & 1.22 & 20.5 & 1.20 & 12.5 & 1.24 & 11.6 & 1.09 \\
\hline n-heptane & 57.3 & 1.27 & 42.9 & 1.27 & 24.5 & 1.33 & 22.2 & 1.15 \\
\hline n-octane & 119.5 & 1.41 & 89.0 & 1.36 & 51.5 & 1.30 & 40.9 & 1.26 \\
\hline n-decane & 484.7 & 1.79 & 360.1 & 1.60 & 179.0 & 1.54 & 129.5 & 1.53 \\
\hline benzene & 69.9 & 0.61 & 54.3 & 0.59 & 31.4 & 0.63 & 28.6 & 0.56 \\
\hline toluene & 157.9 & 0.66 & 118.5 & 0.64 & 63.3 & 0.69 & 56.7 & 0.60 \\
\hline ethylbenzene & 325.1 & 0.71 & 235.0 & 0.72 & 113.3 & 0.78 & 99.0 & 0.68 \\
\hline propylbenzene & 576.6 & 0.84 & 379.3 & 0.88 & 209.9 & 0.81 & 166.7 & 0.76 \\
\hline isopropylbenzene & 466.3 & 0.77 & 315.4 & 0.80 & 173.4 & 0.76 & 137.9 & 0.72 \\
\hline cyclohexane & 47.7 & 0.91 & 39.7 & 0.85 & 22.4 & 0.93 & 21.2 & 0.79 \\
\hline methylcyclohexane & 76.7 & 1.01 & 60.0 & 0.98 & 37.3 & 0.95 & 30.1 & 0.92 \\
\hline o-xylene & 444.0 & 0.67 & 306.9 & 0.68 & 148.4 & 0.75 & 129.8 & 0.64 \\
\hline p-xylene & 341.8 & 0.72 & 240.5 & 0.72 & 122.9 & 0.76 & 110.3 & 0.64 \\
\hline styrene & 434.7 & 0.71 & 320.2 & 0.68 & 169.0 & 0.68 & 134.7 & 0.64 \\
\hline
\end{tabular}

Table 4: Specific retention volume $(\mathrm{ml} / \mathrm{g})$ and activity coefficients at infinite dilution calculated for Squalane at different temperatures

\begin{tabular}{|c|c|c|c|c|c|c|}
\hline \multicolumn{7}{|c|}{ Squalane } \\
\hline \multirow[t]{2}{*}{ Solute } & \multicolumn{2}{|c|}{$\mathrm{T}=80^{\circ} \mathrm{C}$} & \multicolumn{2}{|c|}{$\mathrm{T}=90^{\circ} \mathrm{C}$} & \multicolumn{2}{|c|}{$\mathrm{T}=100^{\circ} \mathrm{C}$} \\
\hline & $\mathrm{V}_{\mathrm{g}}^{\mathbf{0}}$ & $\gamma^{\infty}$ & $\mathbf{V}_{\mathrm{g}}{ }^{0}$ & $\gamma^{\infty}$ & $V_{g}{ }^{0}$ & $\gamma^{\infty}$ \\
\hline n-hexane & 53.4 & 0.76 & 40.5 & 0.77 & 33.0 & 0.74 \\
\hline n-heptane & 124.2 & 0.79 & 90.4 & 0.80 & 70.4 & 0.77 \\
\hline n-octane & 286.6 & 0.82 & 199.6 & 0.86 & 149.0 & 0.80 \\
\hline n-decane & 1526.0 & 0.87 & 917.9 & 0.93 & 636.9 & 0.89 \\
\hline benzene & 78.7 & 0.71 & 57.5 & 0.73 & 48.3 & 0.66 \\
\hline toluene & 195.5 & 0.73 & 140.5 & 0.73 & 109.0 & 0.69 \\
\hline ethylbenzene & 422.3 & 0.77 & 271.8 & 0.83 & 207.3 & 0.78 \\
\hline propylbenzene & 849.9 & 0.84 & 550.2 & 0.87 & 410.3 & 0.80 \\
\hline isopropylbenzene & 672.2 & 0.77 & 439.9 & 0.81 & 331.8 & 0.84 \\
\hline cyclohexane & 95.5 & 0.60 & 71.7 & 0.60 & 57.6 & 0.58 \\
\hline methylcyclohexane & 163.5 & 0.64 & 119.7 & 0.64 & 93.8 & 0.62 \\
\hline o-xylene & 553.5 & 0.77 & 379.4 & 0.77 & 279.8 & 0.74 \\
\hline p-xylene & 462.5 & 0.76 & 307.9 & 0.79 & 237.3 & 0.73 \\
\hline styrene & 513.8 & 0.89 & 345.9 & 0.88 & 262.1 & 0.82 \\
\hline
\end{tabular}


Table 5: Specific retention volume $(\mathrm{ml} / \mathrm{g})$ and activity coefficients at infinite dilution calculated for Carbowax-400 at different temperatures

\begin{tabular}{|c|c|c|c|c|c|c|}
\hline \multicolumn{7}{|c|}{ Carbowax-400 } \\
\hline \multirow[t]{2}{*}{ Solute } & \multicolumn{2}{|c|}{$\mathrm{T}=80^{\circ} \mathrm{C}$} & \multicolumn{2}{|c|}{$\mathrm{T}=90^{\circ} \mathrm{C}$} & \multicolumn{2}{|c|}{$\mathrm{T}=100^{\circ} \mathrm{C}$} \\
\hline & $\mathbf{V}_{\mathrm{g}}^{0}$ & $\gamma^{\infty}$ & $\mathbf{V}_{\mathrm{g}}^{\mathbf{0}}$ & $\gamma^{\infty}$ & $\mathbf{V}_{\mathrm{g}}^{\mathbf{0}}$ & $\gamma^{\infty}$ \\
\hline n-hexane & 4.6 & 9.25 & 3.8 & 8.56 & 3.2 & 8.00 \\
\hline n-heptane & 8.7 & 11.91 & 6.9 & 10.92 & 5.7 & 10.03 \\
\hline n-octane & 16.4 & 15.23 & 12.7 & 13.82 & 10.0 & 12.62 \\
\hline n-nonane & 30.4 & 19.48 & 22.7 & 17.56 & 17.4 & 15.83 \\
\hline n-decane & 56.6 & 24.87 & 40.6 & 22.35 & 30.1 & 20.00 \\
\hline 1-hexene & 6.5 & 5.62 & 5.3 & 5.30 & 4.3 & 5.05 \\
\hline 1-heptene & 12.3 & 7.33 & 9.7 & 6.86 & 7.7 & 6.49 \\
\hline 1-octene & 22.8 & 9.39 & 17.4 & 8.75 & 13.5 & 8.18 \\
\hline 1-nonene & 42.6 & 12.04 & 31.2 & 11.18 & 23.4 & 10.41 \\
\hline 1-decene & 78.3 & 16.42 & 55.5 & 14.23 & 40.4 & 13.10 \\
\hline benzene & 53.2 & 1.11 & 39.0 & 1.13 & 29.2 & 1.15 \\
\hline toluene & 97.2 & 1.54 & 68.2 & 1.59 & 52.4 & 1.52 \\
\hline ethylbenzene & 159.2 & 2.17 & 110.8 & 2.16 & 78.9 & 2.17 \\
\hline propylbenzene & 255.2 & 2.95 & 173.4 & 2.92 & 120.2 & 2.90 \\
\hline isopropylbenzene & 209.4 & 2.63 & 141.8 & 2.65 & 100.0 & 2.63 \\
\hline cyclohexane & 12.2 & 4.95 & 9.7 & 4.71 & 7.8 & 4.49 \\
\hline methylcyclohexane & 16.2 & 6.77 & 12.6 & 6.44 & 10.1 & 6.08 \\
\hline o-xylene & 219.6 & 2.05 & 155.3 & 1.99 & 109.5 & 1.99 \\
\hline m-xylene & 167.0 & 2.29 & 119.6 & 2.21 & 84.3 & 2.22 \\
\hline p-xylene & 161.7 & 2.29 & 115.2 & 2.23 & 82.2 & 2.22 \\
\hline styrene & 344.7 & 1.37 & 236.0 & 1.36 & 159.7 & 1.42 \\
\hline
\end{tabular}

Table 6: Specific retention volume $(\mathrm{ml} / \mathrm{g})$ and activity coefficients at infinite dilution calculated for Carbowax-1500 at different temperatures

\begin{tabular}{|c|c|c|c|c|c|c|}
\hline \multicolumn{7}{|c|}{ Carbowax-1500 } \\
\hline \multirow[t]{2}{*}{ Solute } & \multicolumn{2}{|c|}{$\mathbf{T}=80^{\circ} \mathrm{C}$} & \multicolumn{2}{|c|}{$\mathrm{T}=90^{\circ} \mathrm{C}$} & \multicolumn{2}{|c|}{$\mathrm{T}=100^{\circ} \mathrm{C}$} \\
\hline & $\mathbf{V}_{\mathrm{g}}{ }^{0}$ & $\gamma^{\infty}$ & $\mathbf{V}_{\mathrm{g}}^{0}$ & $\gamma^{\infty}$ & $\mathbf{V}_{\mathrm{g}}^{\mathbf{0}}$ & $\gamma^{\infty}$ \\
\hline n-hexane & 5.3 & 2.14 & 4.2 & 2.04 & 3.5 & 1.95 \\
\hline n-heptane & 10.1 & 2.74 & 8.0 & 2.53 & 6.4 & 2.37 \\
\hline n-octane & 19.4 & 3.43 & 14.7 & 3.18 & 11.2 & 2.99 \\
\hline n-nonane & 33.9 & 4.66 & 25.5 & 4.17 & 18.8 & 3.91 \\
\hline n-decane & 70.0 & 5.36 & 49.0 & 4.94 & 35.1 & 4.58 \\
\hline 1-hexene & 6.9 & 1.42 & 5.7 & 1.32 & 4.6 & 1.28 \\
\hline 1-heptene & 13.3 & 1.81 & 10.4 & 1.70 & 8.0 & 1.17 \\
\hline 1-octene & 24.9 & 2.29 & 19.0 & 2.14 & 14.2 & 2.08 \\
\hline 1-nonene & 46.9 & 2.91 & 34.5 & 2.69 & 24.9 & 2.61 \\
\hline 1-decene & 87.9 & 3.90 & 62.4 & 3.38 & 43.6 & 3.24 \\
\hline benzene & 58.4 & 0.27 & 43.3 & 0.27 & 32.2 & 0.28 \\
\hline toluene & 108.5 & 0.37 & 76.9 & 0.37 & 56.0 & 0.38 \\
\hline ethylbenzene & 180.8 & 0.51 & 126.1 & 0.51 & 89.4 & 0.51 \\
\hline propylbenzene & 273.2 & 0.73 & 197.2 & 0.68 & 139.2 & 0.67 \\
\hline isopropylbenzene & 220.9 & 0.66 & 151.9 & 0.66 & 114.9 & 0.61 \\
\hline cyclohexane & 13.6 & 1.18 & 10.9 & 1.12 & 9.0 & 1.04 \\
\hline methylcyclohexane & 18.5 & 1.56 & 14.7 & 1.47 & 11.8 & 1.38 \\
\hline o-xylene & 261.4 & 0.46 & 177.3 & 0.47 & 124.5 & 0.47 \\
\hline m-xylene & 182.5 & 0.56 & 128.2 & 0.55 & 94.6 & 0.53 \\
\hline p-xylene & 187.7 & 0.52 & 131.6 & 0.52 & 92.4 & 0.53 \\
\hline styrene & 373.2 & 0.34 & 263.8 & 0.32 & 183.5 & 0.33 \\
\hline
\end{tabular}


Table 7: Specific retention volume $(\mathrm{ml} / \mathrm{g})$ and activity coefficients at infinite dilution calculated for Carbowax-4000 at different temperatures

\begin{tabular}{|c|c|c|c|c|c|c|}
\hline \multicolumn{7}{|c|}{ Carbowax-4000 } \\
\hline \multirow[t]{2}{*}{ Solute } & \multicolumn{2}{|c|}{$\mathrm{T}=\mathbf{8 0}^{\circ} \mathrm{C}$} & \multicolumn{2}{|c|}{$\mathrm{T}=90^{\circ} \mathrm{C}$} & \multicolumn{2}{|c|}{$\mathrm{T}=100^{\circ} \mathrm{C}$} \\
\hline & $\mathbf{V}_{\mathrm{g}}^{0}$ & $\gamma^{\infty}$ & $\mathbf{V}_{\mathrm{g}}^{0}$ & $\gamma^{\infty}$ & $\mathrm{V}_{\mathrm{g}}^{0}$ & $\gamma^{\infty}$ \\
\hline n-hexane & 6.2 & 0.68 & 5.1 & 0.64 & 4.1 & 0.63 \\
\hline n-heptane & 12.2 & 0.85 & 9.5 & 0.80 & 7.4 & 0.77 \\
\hline n-octane & 23.5 & 1.06 & 17.8 & 0.98 & 13.4 & 0.94 \\
\hline n-nonane & 45.0 & 1.32 & 32.9 & 1.21 & 24.0 & 1.15 \\
\hline n-decane & 86.0 & 1.64 & 60.6 & 1.49 & 42.9 & 1.40 \\
\hline 1-hexene & 8.5 & 0.43 & 6.8 & 0.41 & 5.5 & 0.40 \\
\hline 1-heptene & 16.5 & 0.54 & 12.5 & 0.53 & 10.0 & 0.50 \\
\hline 1-octene & 31.7 & 0.68 & 23.2 & 0.66 & 17.8 & 0.62 \\
\hline 1-nonene & 60.6 & 0.85 & 43.0 & 0.81 & 31.9 & 0.76 \\
\hline 1-decene & 116.0 & 1.11 & 79.2 & 1.00 & 56.8 & 0.93 \\
\hline benzene & 61.8 & 0.09 & 47.0 & 0.09 & 34.6 & 0.08 \\
\hline toluene & 118.8 & 0.12 & 84.3 & 0.12 & 60.6 & 0.13 \\
\hline ethylbenzene & 196.9 & 0.17 & 141.2 & 0.17 & 98.7 & 0.17 \\
\hline propylbenzene & 325.4 & 0.23 & 229.0 & 0.22 & 154.9 & 0.22 \\
\hline isopropylbenzene & 261.2 & 0.21 & 184.8 & 0.20 & 126.4 & 0.21 \\
\hline cyclohexane & 15.4 & 0.39 & 12.7 & 0.36 & 10.1 & 0.35 \\
\hline methylcyclohexane & 21.1 & 0.52 & 17.1 & 0.47 & 13.3 & 0.46 \\
\hline o-xylene & 278.4 & 0.16 & 197.7 & 0.16 & 136.6 & 0.16 \\
\hline m-xylene & 213.6 & 0.18 & 153.3 & 0.17 & 107.1 & 0.17 \\
\hline p-xylene & 205.4 & 0.18 & 148.9 & 0.17 & 104.0 & 0.17 \\
\hline styrene & 427.7 & 0.11 & 295.4 & 0.11 & 197.2 & 0.11 \\
\hline
\end{tabular}

Table 8: Specific retention volume $(\mathrm{ml} / \mathrm{g})$ and activity coefficients at infinite dilution calculated for Amine220 at different temperatures

\begin{tabular}{|c|c|c|c|c|c|c|}
\hline \multicolumn{7}{|c|}{ Amine-220 } \\
\hline \multirow[t]{2}{*}{ Solute } & \multicolumn{2}{|c|}{$\mathrm{T}=70^{\circ} \mathrm{C}$} & \multicolumn{2}{|c|}{$\mathrm{T}=80^{\circ} \mathrm{C}$} & \multicolumn{2}{|c|}{$\mathrm{T}=90^{\circ} \mathrm{C}$} \\
\hline & $\mathbf{V}_{\mathrm{g}}^{\mathbf{0}}$ & $\gamma^{\infty}$ & $V_{g}{ }^{0}$ & $\gamma^{\infty}$ & $V_{g}^{0}$ & $\gamma^{\infty}$ \\
\hline n-hexane & 30.1 & 2.17 & 23.1 & 2.12 & 17.0 & 2.21 \\
\hline n-heptane & 69.1 & 2.40 & 50.2 & 2.37 & 36.3 & 2.40 \\
\hline n-octane & 156.8 & 2.66 & 109.0 & 2.62 & 74.9 & 2.68 \\
\hline n-nonane & 328.7 & 3.16 & 214.7 & 3.16 & 145.4 & 3.14 \\
\hline n-decane & 786.8 & 3.28 & 503.3 & 3.20 & 315.5 & 3.29 \\
\hline 1-hexene & 28.6 & 1.95 & 21.2 & 1.99 & 16.3 & 1.98 \\
\hline 1-heptene & 65.1 & 2.19 & 46.3 & 2.22 & 34.0 & 2.24 \\
\hline 1-octene & 146.4 & 2.43 & 99.6 & 2.46 & 70.3 & 2.48 \\
\hline 1-nonene & 329.1 & 2.70 & 214.2 & 2.74 & 144.8 & 2.75 \\
\hline 1-decene & 733.1 & 3.20 & 456.2 & 3.22 & 306.9 & 2.94 \\
\hline benzene & 94.2 & 0.98 & 69.9 & 0.96 & 51.0 & 0.99 \\
\hline toluene & 218.1 & 1.12 & 154.9 & 1.11 & 108.9 & 1.14 \\
\hline ethylbenzene & 447.7 & 1.30 & 305.4 & 1.29 & 207.3 & 1.32 \\
\hline propylbenzene & 893.6 & 1.48 & 585.7 & 1.47 & 384.4 & 1.50 \\
\hline isopropylbenzene & 665.8 & 1.42 & 459.8 & 1.37 & 305.3 & 1.41 \\
\hline cyclohexane & 61.9 & 1.51 & 47.1 & 1.47 & 34.9 & 1.50 \\
\hline methylcyclohexane & 101.5 & 1.71 & 75.5 & 1.66 & 53.9 & 1.72 \\
\hline o-xylene & 620.5 & 1.24 & 425.6 & 1.21 & 281.6 & 1.26 \\
\hline m-xylene & 462.3 & 1.40 & 310.2 & 1.40 & 215.4 & 1.40 \\
\hline p-xylene & 486.3 & 1.29 & 335.6 & 1.26 & 223.9 & 1.31 \\
\hline styrene & 708.3 & 1.14 & 487.7 & 1.10 & 323.9 & 1.14 \\
\hline
\end{tabular}


Table 9: Specific retention volume $(\mathrm{ml} / \mathrm{g})$ and activity coefficients at infinite dilution calculated for Tributyl phosphate at different temperatures

\begin{tabular}{|c|c|c|c|c|c|c|}
\hline \multicolumn{7}{|c|}{ Tributyl phosphate } \\
\hline \multirow[t]{2}{*}{ Solute } & \multicolumn{2}{|c|}{$\mathrm{T}=80^{\circ} \mathrm{C}$} & \multicolumn{2}{|c|}{$\mathrm{T}=90^{\circ} \mathrm{C}$} & \multicolumn{2}{|c|}{$\mathrm{T}=100^{\circ} \mathrm{C}$} \\
\hline & $\mathbf{V}_{\mathrm{g}}^{\mathbf{0}}$ & $\gamma^{\infty}$ & $\mathbf{V}_{\mathrm{g}}{ }^{0}$ & $\gamma^{\infty}$ & $\mathbf{V}_{\mathrm{g}}{ }^{\mathrm{T}}$ & $\gamma^{\infty}$ \\
\hline n-hexane & 15.2 & 3.06 & 12.0 & 2.96 & 9.6 & 2.90 \\
\hline n-heptane & 33.8 & 3.75 & 25.1 & 3.31 & 19.3 & 3.22 \\
\hline n-octane & 70.5 & 3.85 & 51.8 & 3.68 & 38.2 & 3.59 \\
\hline n-nonane & 149.4 & 4.21 & 105.8 & 4.11 & 75.3 & 3.99 \\
\hline n-decane & 315.7 & 4.85 & 214.9 & 4.89 & 147.7 & 4.44 \\
\hline 1-hexene & 17.4 & 2.30 & 13.9 & 2.20 & 11.1 & 2.17 \\
\hline 1-heptene & 38.0 & 2.57 & 29.1 & 2.49 & 22.2 & 2.46 \\
\hline 1-octene & 81.2 & 2.88 & 59.8 & 2.77 & 44.0 & 2.73 \\
\hline 1-nonene & 171.1 & 3.26 & 122.4 & 3.10 & 86.9 & 3.05 \\
\hline 1-decene & 363.6 & 3.84 & 248.1 & 3.46 & 169.1 & 3.41 \\
\hline benzene & 74.3 & 0.85 & 56.7 & 0.85 & 42.0 & 0.87 \\
\hline toluene & 165.1 & 0.99 & 120.8 & 0.97 & 86.6 & 1.00 \\
\hline ethylbenzene & 327.6 & 1.14 & 232.0 & 1.12 & 160.4 & 1.16 \\
\hline propylbenzene & 620.4 & 1.32 & 426.5 & 1.29 & 286.0 & 1.32 \\
\hline isopropylbenzene & 497.1 & 1.20 & 347.9 & 1.17 & 234.2 & 1.22 \\
\hline cyclohexane & 32.7 & 2.01 & 25.8 & 1.92 & 20.2 & 1.88 \\
\hline methylcyclohexane & 51.5 & 2.31 & 40.3 & 2.19 & 30.5 & 2.18 \\
\hline o-xylene & 459.3 & 1.07 & 317.6 & 1.06 & 218.0 & 1.09 \\
\hline m-xylene & 360.8 & 1.15 & 253.4 & 1.13 & 175.4 & 1.16 \\
\hline p-xylene & 352.7 & 1.14 & 244.2 & 1.14 & 172.3 & 1.15 \\
\hline styrene & 576.8 & 0.89 & 397.9 & 0.88 & 269.2 & 0.91 \\
\hline
\end{tabular}

Table 10: Specific retention volume $(\mathrm{ml} / \mathrm{g})$ and activity coefficients at infinite dilution calculated for Trixylenil phosphate at different temperatures

\begin{tabular}{|c|c|c|c|c|c|c|}
\hline \multicolumn{7}{|c|}{ Trixylenil phosphate } \\
\hline \multirow[t]{2}{*}{ Solute } & \multicolumn{2}{|c|}{$\mathrm{T}=80^{\circ} \mathrm{C}$} & \multicolumn{2}{|c|}{$\mathrm{T}=90^{\circ} \mathrm{C}$} & \multicolumn{2}{|c|}{$\mathrm{T}=100^{\circ} \mathrm{C}$} \\
\hline & $\mathbf{V}_{\mathrm{g}}^{0}$ & $\gamma^{\infty}$ & $\mathbf{V}_{\mathrm{g}}^{0}$ & $\gamma^{\infty}$ & $\mathbf{V}_{\mathrm{g}}^{\mathbf{0}}$ & $\gamma^{\infty}$ \\
\hline n-hexane & 16.4 & 2.54 & 12.3 & 2.60 & 10.1 & 2.47 \\
\hline n-heptane & 35.7 & 2.84 & 25.8 & 2.89 & 20.6 & 2.71 \\
\hline n-octane & 76.6 & 3.18 & 55.1 & 3.11 & 41.2 & 2.99 \\
\hline n-nonane & 163.8 & 3.53 & 113.0 & 3.45 & 81.3 & 3.32 \\
\hline n-decane & 348.4 & 3.94 & 231.4 & 3.82 & 159.7 & 3.68 \\
\hline 1-hexene & 18.3 & 1.97 & 13.6 & 2.02 & 11.0 & 1.95 \\
\hline 1-heptene & 40.4 & 2.17 & 28.6 & 2.27 & 22.8 & 2.15 \\
\hline 1-octene & 86.6 & 2.42 & 59.1 & 2.52 & 45.8 & 2.36 \\
\hline 1-nonene & 185.7 & 2.69 & 121.9 & 2.79 & 92.5 & 2.57 \\
\hline 1-decene & 393.8 & 3.19 & 248.0 & 3.11 & 180.5 & 2.87 \\
\hline benzene & 77.8 & 0.74 & 58.2 & 0.74 & 43.5 & 0.76 \\
\hline toluene & 174.6 & 0.84 & 124.7 & 0.85 & 90.4 & 0.86 \\
\hline ethylbenzene & 348.9 & 0.96 & 240.2 & 0.97 & 168.2 & 0.99 \\
\hline propylbenzene & 667.9 & 1.10 & 445.6 & 1.11 & 301.8 & 1.13 \\
\hline isopropylbenzene & 516.1 & 1.04 & 351.3 & 1.04 & 245.4 & 1.05 \\
\hline cyclohexane & 35.1 & 1.68 & 27.7 & 1.61 & 21.3 & 1.61 \\
\hline methylcyclohexane & 55.9 & 1.92 & 42.1 & 1.88 & 32.2 & 1.86 \\
\hline o-xylene & 476.7 & 0.92 & 324.2 & 0.93 & 224.5 & 0.95 \\
\hline m-xylene & 373.8 & 1.00 & 258.7 & 1.00 & 183.5 & 1.00 \\
\hline p-xylene & 362.3 & 1.00 & 253.1 & 0.99 & 176.6 & 1.00 \\
\hline styrene & 602.1 & 0.76 & 404.3 & 0.78 & 276.6 & 0.80 \\
\hline
\end{tabular}




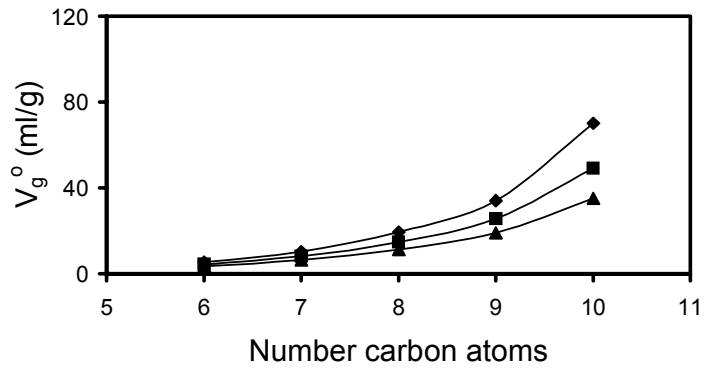

(a)

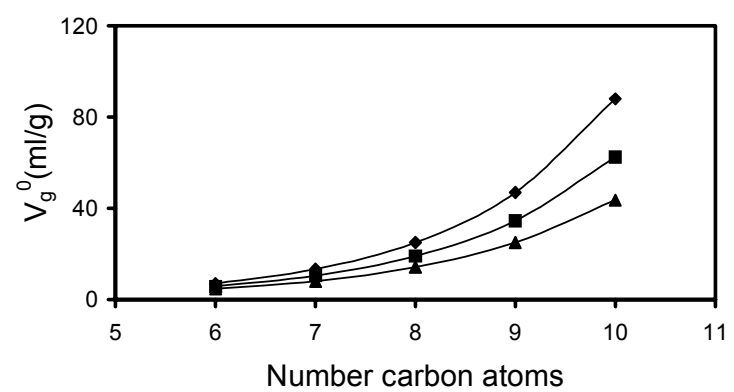

(b)

Figure 1: Specific retention volume on Carbowax-1500 for (a) n-alkanes and (b) alkenes at different temperatures: $80^{\circ} \mathrm{C}(\bullet), 90^{\circ} \mathrm{C}(\boldsymbol{\square}), 100^{\circ} \mathrm{C}(\boldsymbol{\Delta})$

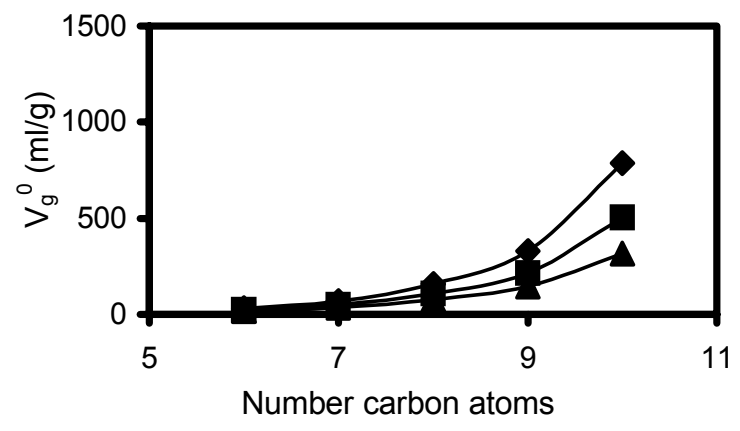

(a)

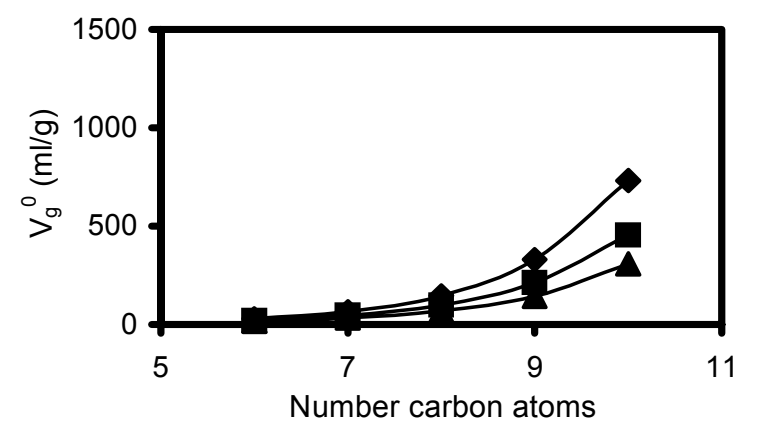

(b)

Figure 2: Specific retention volume on Amine-220 for (a) n-alkanes and (b) alkenes at different temperatures:

$$
70{ }^{\circ} \mathrm{C}(\bullet), 80{ }^{\circ} \mathrm{C}(\boldsymbol{\bullet}), 90^{\circ} \mathrm{C}(\boldsymbol{\Delta})
$$

For the aromatic compounds (Fig. 3 and 4), a more similar behaviour between highly polar and lowly polar liquid phases was found, suggesting a selective interaction of the aromatic with the polar groups of the liquid phase. In the case of cycloalkanes, this selective interaction was lower (the difference in the interaction strength increases again). In both cases, the presence of methyl groups increased the interaction of the solutes with the active phases. This increase was more important for aromatic compounds and was supposedly caused by the increase in the electronic density, and hence the polarity, of the aromatic compounds.

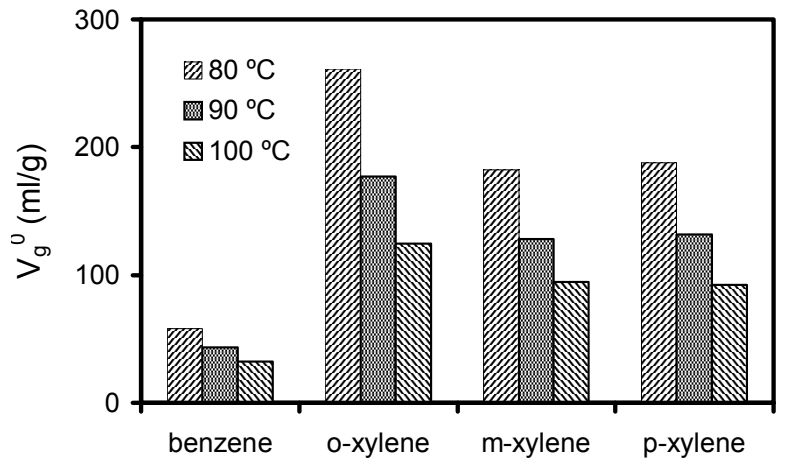

(a)

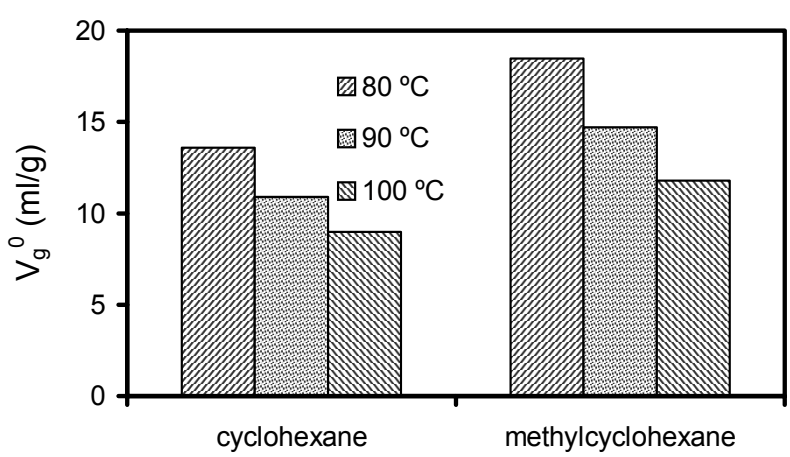

(b)

Figure 3: Specific retention volume on Carbowax-1500 for (a) aromatic and (b) cyclic compounds: 


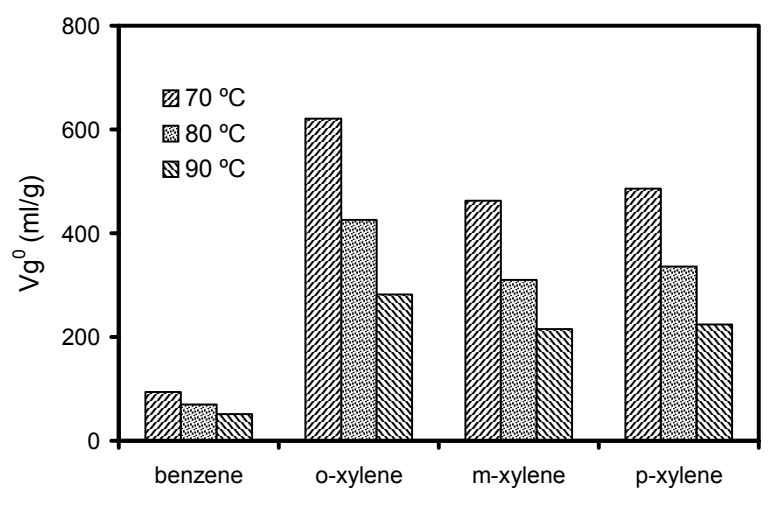

(a)

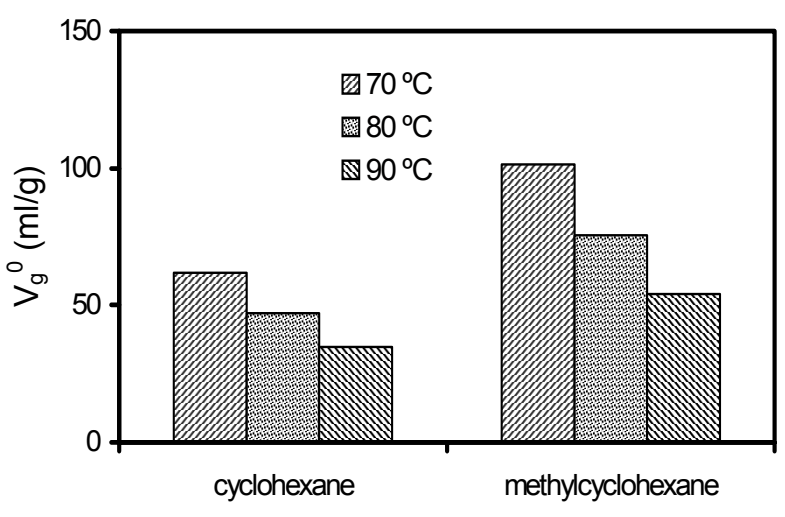

(b)

Figure 4: Specific retention volume on Amine-220 for (a) aromatic and (b) cyclic compounds

Activity coefficients at infinite dilution for squalane were less than one; these low values suggest low solution enthalpies for the methyl-alkane interactions, as found by Langer et al. (1982). It can also be observed that this value increased as the size of solute increased. This can be explained by the parallel arrangement between the solute and the solvent, so small molecules were closer to the solvent, thus having stronger interactions than larger molecules. For the polar liquid phases, it can be observed that alkenes and especially aromatic hydrocarbons were retained with higher selectivity than alkanes, this behaviour being explained by the fact that alkanes experience less polarization. On the other hand, it can also be observed that the solubility of alkanes decreased as the molecular mass of the liquid phase (Carbowax) increased, with this effect being more marked for aromatic compounds than for alkanes and alkenes (Tables 5-7). Due to the electronegativity of the $\mathrm{O}$ atom, the $\mathrm{C}-\mathrm{O}$ bond has a dipole moment, and this polarity is determined by the conformations adopted by chain segments. The global structure of the chain is correlated with polar interactions with other molecules, the strength of the bond between liquid phase and solute increasing as the freedom of the C-O bond increases (González et al., 2002; González and Pérez-Parajon, 2003).

Values of interaction parameter at infinite dilution, $\chi^{\infty}$, obtained with Eq. (5) (Table 11) as well as values of solubility parameter of solute, $\delta_{1}$, obtained with Eq. (10) were used in Eq. (11) to calculate $\delta_{2}$. It had already been established that, when $\chi^{\infty}$ was lower than about 0.5 , the liquid phase and the solute were compatible, whereas when $\chi^{\infty}$ was greater than about 0.5 , solvent-solute interactions were unfavourable (Abraham et al.,
1999). The solubility parameter of the stationary phase, $\delta_{2}$, was determined from the slope of a straight line obtained by plotting the left-hand side of Eq. (11) versus $\delta_{1}$; an illustration of the method employed is shown in Fig. 5 for Squalane. The solubility parameters, $\delta_{2}$, for Squalane, Carbowax400, Carbowax-1500, Carbowax-4000 and Amine220 are listed in Table 12.

According to Eq. (12), probes that interact only with dispersion intermolecular forces (i.e. n-alkanes) should give a straight linear relationship when plotted in accordance with Eq. (11) (Fig. 5). However, probes that have other contributions to their retention will deviate from the line, with the degree of deviation being a measure of the strength of the polar components. Typical plots are depicted in Fig. 6, with the difference between the n-alkanes and alkenes being clear in these plots. The contributions to $\delta_{2}$ for the compounds studied are given in Table 12.

Although values of the specific retention volumes are found in the literature, solubility parameters are not often reported. To obtain the specific retention volume dependence on the solubility parameters for the systems studied here, values of $\mathrm{V}_{\mathrm{g}}{ }^{0}$ for a solute are plotted vs. values of $\tau$ for the different liquid phases studied in this work. As can be observed in Fig. 7, an exponential trend can be inferred from these plots, so these plots can be linearized by an equation of the following type:

$$
\mathrm{V}_{\mathrm{g}}^{0}=\mathrm{a} \ln \tau+\mathrm{b}
$$

where $\mathrm{a}$ and $\mathrm{b}$ are parameters for each hydrocarbon and are linear functions of the number of carbon 
atoms, $\mathrm{N}$, within a homologous series (correlation coefficient, $\mathrm{r}^{2}$, of 0.996 and 0.98 for alkanes and alkenes, respectively). The equations for parameters $\mathrm{a}$ and $\mathrm{b}$ are shown in Table 13.

An examination of Eq. (13) may give indications about the retention of a solute in a liquid phase and some conclusions can be drawn: (i) A solute will have approximately the same retention volume in any non-polar liquid phase and (ii) a solute will have approximately the same retention volume in any liquid phase with the same polar solubility parameter. Hence, the polar solubility parameter $\tau_{1}$ is a useful parameter for classifying and selecting liquid phases for a given separation.

Table 11: Interaction parameters, $\chi^{\infty}$, of Squalane, Carbowax-400, Carbowax-1500, Carbowax-4000 and Amine-220 with some n-alkanes and alkenes at $80^{\circ} \mathrm{C}$

\begin{tabular}{|l|c|c|c|c|c|}
\hline \multicolumn{1}{|c|}{ Hydrocarbon } & Squalane & Carbowax 400 & Carbowax 1500 & Carbowax 4000 & Amine 220 \\
\hline n-hexane & 0.66 & 3.17 & 2.69 & 1.66 & -0.03 \\
n-heptane & 0.40 & 3.65 & 3.22 & 2.05 & 0.31 \\
n-octane & 0.16 & 4.16 & 3.80 & 2.48 & 0.66 \\
n-nonane & 0.07 & 4.61 & 4.27 & 2.80 & 0.86 \\
n-decane & -0.03 & 5.10 & 4.77 & 3.16 & 1.09 \\
1-hexene & 0.45 & 2.60 & 2.29 & 1.18 & 5.77 \\
1-heptene & 0.20 & 3.27 & 2.85 & 1.65 & 6.95 \\
1-octene & -0.02 & 3.76 & 3.43 & 2.14 & 8.14 \\
1-nonene & -0.30 & 4.83 & 4.16 & 2.80 & 9.54 \\
1-decene & -0.40 & 5.41 & 4.65 & 3.18 & 10.58 \\
\hline
\end{tabular}

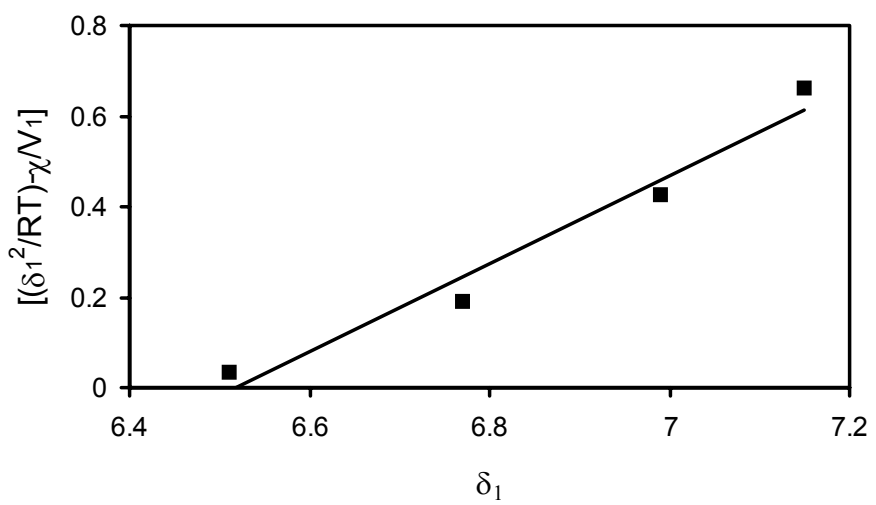

Figure 5: Variation in term $\left[\delta_{1}{ }^{2} / \mathrm{RT}-\chi_{12}{ }^{\infty} / \mathrm{V}_{1}\right]$ with solubility parameters of solutes, $\delta_{1}\left(\mathrm{cal} / \mathrm{cm}^{3}\right)^{0.5}$ at $80^{\circ} \mathrm{C}$ for Squalane (see Eq. 11$)$

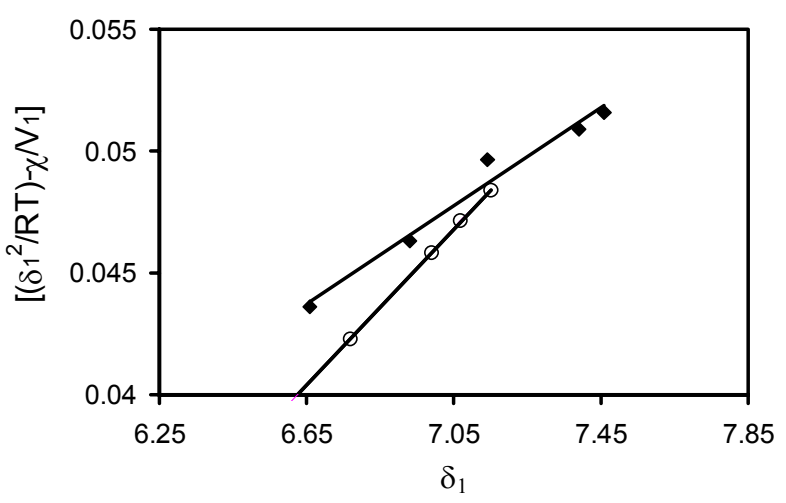

(a)

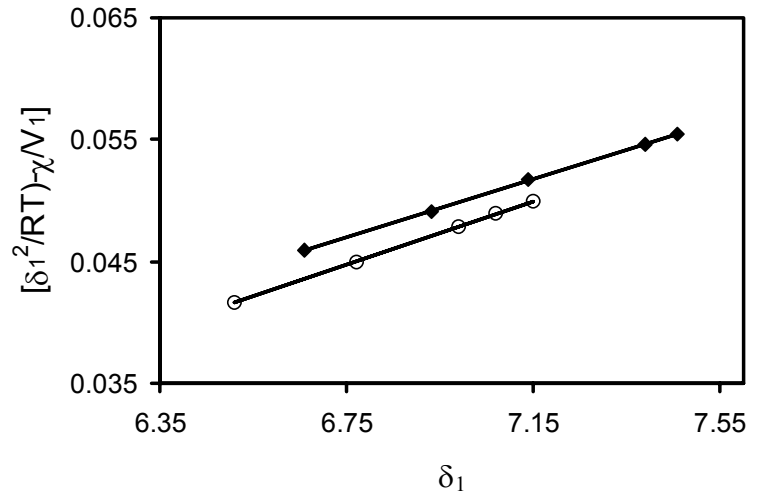

(b) 


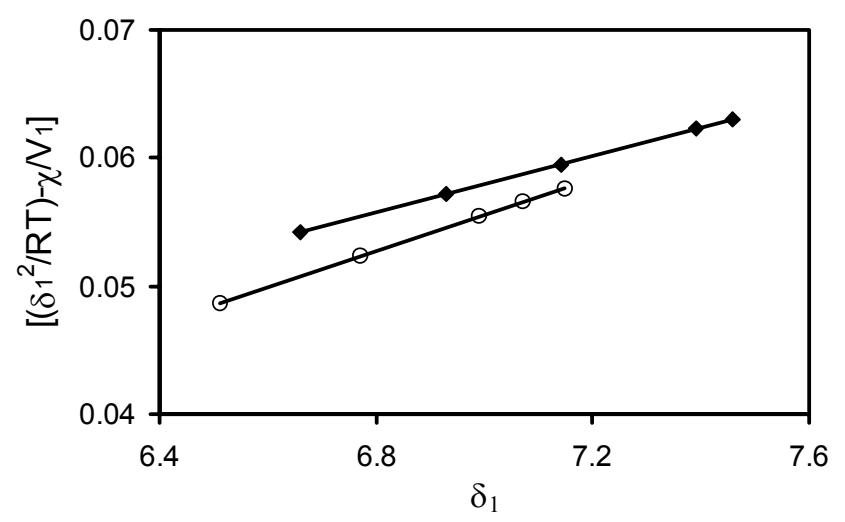

(c)

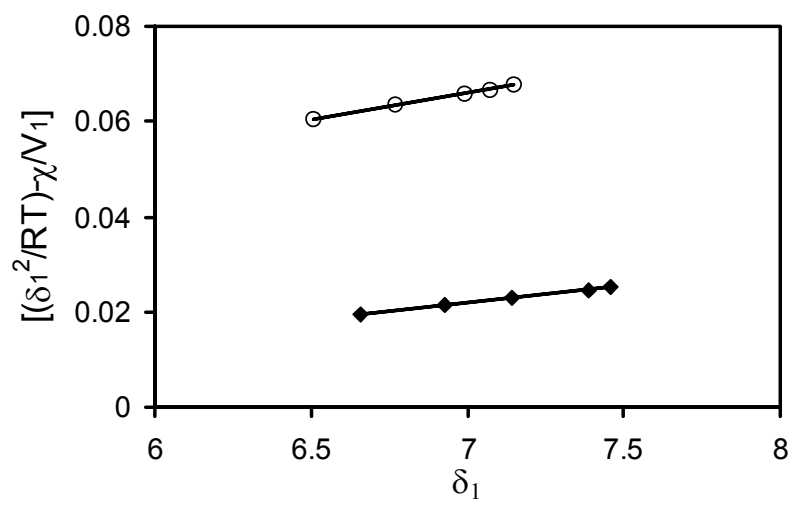

(d)

Figure 6: Estimation of the contributions to the solubility parameter at $80^{\circ} \mathrm{C}$ [alkanes ( $\bullet$ ), alkenes (o)] for different liquid phases: (a) Carbowax 400, (b) Carbowax 1500, (c) Carbowax 4000, (d) Amine 220.

Table 12: Solubility parameter, $\delta_{2}\left(\mathrm{cal} / \mathrm{cm}^{3}\right)^{0.5}$, and the dispersive, $\lambda$, and polar, $\tau$, contributions at $80^{\circ} \mathrm{C}$ for different liquid phases

\begin{tabular}{|l|c|c|c|}
\hline \multicolumn{1}{|c|}{ Liquid phase } & $\boldsymbol{\delta}_{\mathbf{2}}$ & $\boldsymbol{\lambda}$ & $\boldsymbol{\tau}$ \\
\hline Squalane & 9.19 & 9.19 & 3.82 \\
Carbowax-400 & 6.90 & 5.75 & 4.53 \\
Carbowax-1500 & 6.40 & 4.52 & 3.90 \\
Carbowax-4000 & 6.27 & 4.90 & 3.46 \\
Amine-220 & 5.16 & 3.83 & \\
\hline
\end{tabular}

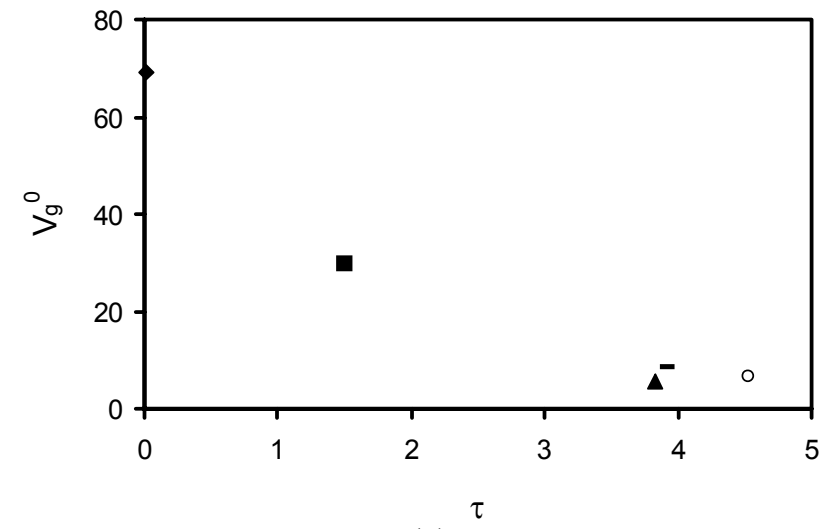

(a)

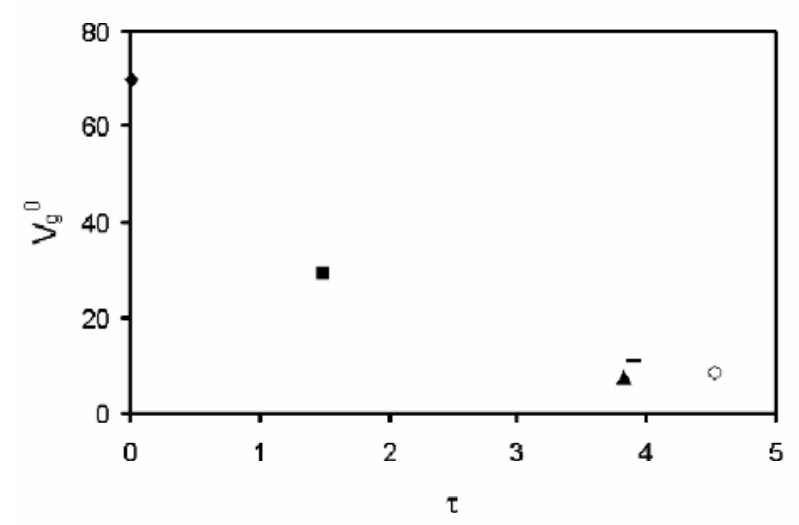

(b)

Figure 7: $\mathrm{V}_{\mathrm{g}}{ }^{0}$ as a function of $\tau$ at $80^{\circ} \mathrm{C}$ for different liquid phases [Squalane ( $\left.\bullet\right)$, Amine $220(\mathbf{\square})$, Carbowax 400 ( $\Delta$ ), Carbowax 1500 (०), Carbowax 4000 (-)] : (a) for n-hexane, (b) for 1-hexene

Table 13: Relationship between the coefficients of Eq. (13) and the number of atoms $(\mathrm{N})$ of alkanes and alkenes.

\begin{tabular}{|l|l|}
\hline normal alkane series & $\begin{array}{l}\mathrm{a}=-73 \mathrm{~N}+465 \\
\mathrm{~b}=156 \mathrm{~N}-981\end{array}$ \\
\hline 1-alkene series & $\begin{array}{l}\mathrm{a}=-193 \mathrm{~N}+1234 \\
\mathrm{~b}=300 \mathrm{~N}-1905\end{array}$ \\
\hline
\end{tabular}




\section{CONCLUSIONS}

Retention data obtained by gas chromatography was used successfully for calculating the polar solubility parameter of a given liquid phase. Results obtained for different liquid phases and solutes were compared in terms of specific retention volumes and activity coefficients.

This work shows that values of solubility parameter, $\delta$, involving the polar and apolar contributions ( $\tau$ and $\lambda$, respectively) can be estimated from specific retention volume, $\mathrm{V}_{\mathrm{g}}{ }^{0}$. This parameter is related with the relative volatility, which determines the distribution of components between different phases (Everson and Van der Merwe, 1998).

\section{REFERENCES}

Abraham, H., Poole, C.F. and Poole, S.K., Classification of Stationary Phases and Other Materials by Gas Chromatography, J. Chromatogr. A, 842, 79 (1999).

Alessi, P., Kikic, I., Papo, A. and Torriano, G., Gas chromatographic Characterization of Phosphate Esters, J. Chem. Eng. Data, 23, 29 (1978).

Conder, J.R., The Derivation of Thermodynamic Information from Asymmetrical Chromatographic Peaks when More than One Distribution Mechanism Contributes to Retention, J. Chromatogr. A, 39, 273 (1969).

Deal, C.H. and Derr, E.L., Selectivity and Solvency in Aromatics Recovery, Ind. Eng. Chem. Process Des. Dev., 3, 394 (1964).

ESDU International Ltd - Engineering Sciences Data, Physical Data, General,

November, 1987.

Everson, R.C. and Van der Merwe, B.J., The Effects of Selected Solvents on the Relative Volatility of a Binary System Consisting of 1-Octene and 2Hexanone, Fluid Phase Equilibria, 143, 173 (1998).

González, F.R. and Pérez-Parajón, J., Effects of Solvent Density on Retention in Gas - Liquid chromatography: II. Polar Solutes in Poly(ethylene glycol) Stationary Phases, J. Chromatogr. A, 969, 265 (2003).

González, F.R., Pérez-Parajón, J. and GarcíaDomínguez, J.A., Effects of Solvent Density on
Retention in Gas Liquid Chromatography: I. Alkanes Solutes in Polyethylene Glycol Stationary Phases, J. Chromatogr. A, 953, 151 (2002).

Hamedi, M. and Danner, R.P., Prediction of Solubility Parameters using the Group Contribution Lattice - Fluid Theory, J. Appl. Polym. Sci., 80, 197 (2001).

Hildebrand, J.H. and Scott, R.L., Regular Solutions, Prentice-Hall, Englewood Cliffs, New York (1962).

Kaya, I., Ilter, Z. and Senol, D., Thermodynamic Interactions and Characterisation of Poly[(glycidyl methacrylate-co-methyl, ethyl, butyl) methacrylate] by Inverse Gas Chromatography, Polymer, 43, 6455 (2002).

Langer, S.H., Sheehan, R.J. and Huang, J.C., Gas Chromatographic Study of the Solution Thermodynamics of Hydroxylic Derivates and Related Compounds, J. Phys. Chem., 86, 4605 (1982).

Letcher, T.M., Harris, R.A., Ramjugernath, D. and Raal, J.D., Activity Coefficients of Hydrocarbon Solutes at Infinite Dilution in Monoethanolamine from Gas - Liquid Chromatography, J. Chem. Thermodynamics, 33, 1655 (2001).

Letcher, T.M., Kozlowska, M.K. and DomanskaZelazna, U., The Determination of Activity Coefficients at Infinite Dilution using G.L.C. for Hydrocarbons in Furfural at $\mathrm{T}=278.15 \mathrm{~K}$ and $\mathrm{T}=298.15 \mathrm{~K}$, J. Chem. Thermodynamics, 36, 37 (2004).

Liu, Z., Zou, H.F., Ye, M.L., Ni, J.Y. and Zhang, Y.K., Effects of Organic Modifiers on Retention Mechanism and Selectivity in Micellar Electrokinetic Capillary Chromatography Studied by Linear Solvation Energy Relationships, J. Chromatogr. A, 863, 69 (1999).

Pena, M.A., Daali, Y., Barra, J. and Bustamante, P., Partial Solubility Parameters of Lactose, Mannitol and Saccharose using the Modified Extended Method and Evaporative Light Scattering Detection, Chem. Pharm. Bull., 48, 179 (2000).

Vega, A. and Coca, J., Activity Coefficients at Infinite Dilution Determined by Gas - Liquid Chromatography: Organic Solvents in Apiezon L, J. Chromatogr. A, 586, 303 (1991).

Vega, A., Díez, F., Esteban, R. and Coca, J., Solvent Selection for Cyclohexane - Cyclohexene Benzene Separation by Extractive Distillation 
using Non-Steady - State Gas Chromatography, Ind. Eng. Chem. Res., 36, 803 (1997).

Voelkel, A. and Kopczynski, T., Inverse Gas Chromatography in the Examination of Organic Compounds: Polarity and Solubility Parameters of Isoquinoline Derivatives, J. Chromatogr. A,
795, 349 (1998).

Weidlich, U., Röhm, H.-J. and Gmehling, J., Measurement of $\gamma^{\infty}$ Using GLC, Part II Results for the Stationary Phases N-Formylmorpholine (NFM) and N-Methylpyrrolidone (NMP), J. Chem. Eng. Data, 32, 450 (1987). 